\title{
Cholangiocarcinoma in Young Cats
}

\author{
Mariana Palha de Brito Jardim 1', Gabriela de Carvalho Cid², Mariana Correia Oliveira' ${ }^{2}$, Rômulo Ferreira de Assumção ${ }^{3}$, \\ Mariana Junger Bastos' ${ }^{1}$, Sheila Lima e Silva ${ }^{1}$, Vivian de Assunção Nogueira ${ }^{4}$ \& Heloisa Justen Moreira de Souza ${ }^{5}$
}

\begin{abstract}
Background: Cholangiocellular carcinoma is a malignant tumor that occurs mainly in elderly dogs and cats. Primary hepatobiliary neoplasms are considered rare in felines, although cholangiocarcinoma is the most common tumor type among malignant liver tumors in the species. The objective of this work is to report two cases of cholangiocarcinoma in young domestic cats, with three years old, and to approach clinical and pathological aspects to promote awareness of this type of injury in young animals, with a focus on prevention of predisposing factors.

Cases: A 3-year-old male domestic cat (cat 1) had a history of progressive weight loss, persistent vomiting, diarrhea, and episodes of seizure. At the clinical examination was noted moderate degree of jaundice. In the biochemical exams it was verified as alteration hypoproteinemia, increase of urea and hyponatremia. Abdominal ultrasonography revealed a formation in the left epigastric region measuring $8.0 \times 5.0 \mathrm{~cm}$, with a diffusely heterogeneous parenchyma, suggestive of hepatic neoplasia, in addition to the presence of free abdominal fluid. Cavity fluid analysis was suggestive of carcinoma. A 3-year-old male domestic cat (cat 2), with history of hyporexia, emaciation and vomiting was taken to care. Physical examination showed moderate dehydration, hypocorous mucosa and presence of abdominal mass on palpation. The animal's serum biochemical assessment were verified as changes, increasing AST. Ultrasonographic examination showed an abdominal mass, located caudally to the spleen, with irregular shape, measuring approximately $4.7 \mathrm{~cm}$ suggestive of hepatic neoplasia, in addition to the presence of abdominal fluid. The hepatic cytology performed by fine needle aspiration was suggestive of carcinoma. After the clinical worsening of two cats and due unfavorable prognosis, euthanasia and necropsy were performed. In the macroscopic analysis of cat 1, numerous nodules of whitish and sometimes "umbilicate" coloration were observed in the liver, measuring up to three $\mathrm{cm}$ in diameter, distributed randomly by the hepatic parenchyma and with a soft consistency at cut. An ovoid-wicking mass, measuring 6.0 x $7.0 \times 5.0 \mathrm{~cm}$ and coloring ranging from white to light brown was checked in omentum. In the macroscopic evaluation of cat 2 , numerous coalescing masses of whitish coloring measuring up to $6.0 \mathrm{~cm}$ in diameter were observed covering almost every hepatic parenchyma and also affecting the pancreas, with a soft consistency at cut. The microscopic findings of both cats were compatible with the diagnosis of cholangiocarcinoma. Considering the immunohistochemical profile, in both cases there was positivity for the CK Pan antibody and CEA and negativity for the CK20, TTF-1, Hepatocyte specific antigen, CD10 and CD56 antibodies. Discussion: Cholangiocarcinomas usually occur in elderly animals, with a mean age of 9 years, so it may be suggested that the cause of the disease in cat 1 and 2 was possibly related to the early and continuous presence of carcinogens. The biliary duct trematod Platynosomum fastosum, the chemical agents plutonium and americium, intestinal parasites such as Ancylostoma spp. and Trichuris vulpis, as well as the occurrence of chronic inflammations independent of etiology are commonly involved in the occurrence of cholangiocarcinoma. Clinical and anatomopathological observations made evident that these two young cats, with three years old, had metastatic cholangiocarcinoma. Thus, veterinary providers should be aware that this tumor type can occur in young animals and is mindful of means of prevention and / or recommended treatment of predisposing factors.
\end{abstract}

Keywords: feline, hepatic neoplasm, cholangiocellular carcinoma. nary Medicine and Surgery, Veterinary Institute, Federal Rural University of Rio de Janeiro (UFRRJ), Seropédica, RJ, Brazil. ${ }^{3}$ Department of General Surgery, State University of Rio de Janeiro (UERJ), Rio de Janeiro, RJ. CORRESPONDENCE: M.P.B. Jardim [jardim.marii@gmail.com - Tel: +55 (21) 9 9318-7447]. Setor de Clínica Médica dos Gatos Domésticos, Instituto de Veterinária, UFRRJ. Rodovia BR 465, Km 07, s/n. Zona Rural. CEP 238990-000 Seropédica, RJ, Brazil. 


\section{INTRODUCTION}

Cholangiocarcinoma are malignant neoplas$\mathrm{ms}$ that primarily originate from the intrahepatic or extrahepatic areas or gallbladder. This tumor type, although widely reported in animal species such as canines, felines, cattle, horses, sheep, and goats, occurs mainly in senior dogs and cats [7]. Primary hepatobiliary tumors are considered rare in cats, with prevalence ranging from $1.5 \%$ to $2.3 \%$ among neoplasms in felines. Bile duct tumors are predominant when compared with hepatocellular tumors and thus constitute the most common type of malignant hepatic neoplasms in cats $[8,11]$. The clinical signs of injury are considered non-specific, and the causes of cholangiocarcinoma are not defined in domestic animals. Some risk factors are predisposing, such as bile and intestinal parasitosis, exposure to chemical agents, and chronic inflammation [6,7]. Cholangiocellular carcinomas are considered intractable due to the potential of dissemination, migration, and progression. Thus, surgical intervention is rarely employed because of the risk of death and relapses and the chemotherapy is generally ineffective $[6,8]$.

The objective of this work is to report two cases of cholangiocarcinoma in young domestic cats, with three years old, and to approach clinical and pathological aspects to promote awareness of this type of injury in young animals, with a focus on prevention of predisposing factors.

\section{CASES}

Cat 1. A 3-year-old male of undefined breed treated at a private clinic in Rio de Janeiro, Brazil. He had a history of progressive weight loss, persistent vomiting, diarrhea, and seizure episodes. During examination, was noticed a moderate degree of jaundice. Serum biochemistry revealed the following: hypoproteinemia, $4.8 \mathrm{mg} / \mathrm{dL}$ (reference values, 5.4 to $7.8 \mathrm{mg} / \mathrm{dL}$ ); high urea, $97.9 \mathrm{mg} / \mathrm{dL}$ (reference values, 30.0 to $60.0 \mathrm{mg} / \mathrm{dL}$ ); and hyponatremia, $130.0 \mathrm{mEq} / \mathrm{L}$ (reference values, 140.0 to $150.0 \mathrm{mEq} / \mathrm{L}$ ). Abdominal ultrasonography showed a formation in the left epigastric region measuring $8.0 \times 5.0 \mathrm{~cm}$, with diffusely heterogeneous parenchyma, suggestive of hepatic neoplasia and abdominal effusion. The abdominal cavity fluid had a cloudy appearance and pink coloration, and red blood cells, non-degenerated neutrophils, activated macrophages, lymphocytes, and rare plasma cells were present. There were also different cell shapes, varying from rounded to oval, with discrete anisocytosis and anisokaryosis.

Cat 2. Also a 3-year-old male of undefined breed treated at the Medical Clinic of Domestic Cats in the Veterinary Hospital of Small Animals of the Federal Rural University of Rio de Janeiro (UFRRJ). He had a history of hyporexia, weight loss, and vomiting. Physical examination of the patient indicated moderate dehydration, pale mucosa, and the presence of an abdominal mass upon palpation. Biochemical evaluation showed an increase in aspartate aminotransferase (AST) levels (290 U/L; reference values, 5.0 to $60 \mathrm{U} / \mathrm{L})$. Ultrasound revealed an abdominal mass located caudally to the spleen with an irregular shape and measuring approximately $4.7 \mathrm{~cm}$; this observation suggested hepatic neoplasm. In addition, abdominal fluid was present. The cytology hepatic was performed via fine needle puncture guided by ultrasonography, where the presence of epithelial cells with moderate cellularity, arranged in groups, with moderate pleomorphism, basaloid appearance, and anisocytosis was noted. The cytoplasm of these cells had well-defined limits and bluish coloration. The nucleus had moderate pleomorphism, anisokaryosis, multinucleation, multiple nucleoli, and moderately coarse chromatin. There were also inflammatory cells with the predominance of neutrophils, mononuclear cells, activated macrophages, and a moderate amount of red blood cells. The cytological analysis suggested carcinoma.

Cats 1 and 2 were both negative for feline leukemia and feline immunodeficiency viruses. In two cases, the initial treatment recommended the control of emesis and support care. After clinical worsening and due unfavorable prognosis, euthanasia and necropsies were performed for both cats.

In cat 1 , numerous, umbilicated nodules of whitish color were observed in the liver, measuring up to $3.0 \mathrm{~cm}$ in diameter and distributed randomly by the parenchyma (Figure 1A), with a soft consistency at the cut. A mass involving the omentum, measuring $6.0 \times 7.0 \times 5.0 \mathrm{~cm}$ and coloring varying from white to light brown, was also checked. In cat 2, numerous coalescing masses of whitish coloring measuring up to $6.0 \mathrm{~cm}$ in diameter were observed covering almost every hepatic parenchyma (Figure 1B), which also affected the pancreas, with a soft consistency at the cut. 
For both patients, organ fragments were collected, fixed in $10 \%$ formalin, and sent to the UFRRJ Pathology Department for histopathological analysis. Specimens were cleaved, processed, and submitted for hematoxylin and eosin (HE) staining and microscopic evaluation.

Histopathological examination of both animals revealed diffuse and marked proliferation of neoplastic cells of epithelial origin, arranged in a tubular form, in the hepatic tissue. Cellular pleomorphism, anisocytosis, anisokaryosis, eosinophilic cytoplasm, nuclei with coarse chromatin, multiple nucleoli, and high mitotic activity were also observed (Figure 2A and Figure 2B). Bands of connective tissue were observed around cellular proliferation, sometimes forming nodules encircled by a discrete lymphoplasmacytic inflammatory infiltrate. Extensive areas of tumor necrosis and hemorrhage were observed. The mass that covered the omentum in cat 1 (Figure 2C) and the mass covering the pancreas (Figure 2D) and lymph node in cat 2 presented neoplastic cell proliferation of epithelial origin similar to those observed in hepatic parenchyma. The microscopic lesions of both felines were compatible with the diagnosis of cholangiocarcinoma.

For immunohistochemistry, tumor fragments from these two animals were embedded in paraffin and sent to the Vetmol laboratory, located in São Paulo, Brazil. Both cases had the same immunohistochemical profile regarding positive and negative tumor cell markers, from which it was possible to confirm the diagnosis of cholangiocarcinoma. The antibodies used and the results of each animal are summarized in Table 1. Figure 3 are photomicrographs for both patients. To note, for the CK7 antibody, a new evaluation was performed in the immunohistochemistry laboratory of the Federal Rural University of Rio de Janeiro, obtaining negative results for each patient.

\section{DISCUSSION}

The clinical signs of cats with hepatobiliary neoplasms include apathy, lethargy, weakness, hyporexia, anorexia, weight loss, vomiting, diarrhea, polydipsia, ascites, abdominal distention, jaundice, and dyspnea [2,5,6,8].

Cats with malignant neoplasms are more prone to show such clinical manifestations than felines with benign tumors [3]. The clinical observations seen in both patients with cholangiocarcinoma corroborate these previous findings.
Hematological and biochemical results indicated abnormal values and alterations in cats 1 and 2; however, any changes in blood exams of cats with bile duct neoplasm are not specific to hepatic tumors, given that some of these alterations may reflect only hepatocellular or biliary stasis [5]. The most common hematological alteration of felines with hepatobiliary neoplasm is leukocytosis. Serum biochemistry showed an increase in alanine aminotransferase, AST, and bilirubin, which are usually higher in cats with malignant hepatic tumors, but these alterations cannot be used as a differential diagnosis of benign hepatic neoplasm. There is also the occurrence of azotemia [3].

Cholangiocarcinoma usually occurs in older animals, with an average age of 9 years $[3,5,8]$. Occurrence in younger animals may be due to feline leukemia virus [6]. However, our patients tested negative for the virus. Thus, occurrence of cholangiocarcinoma in cats 1 and 2 is possibly linked to the early and continuous exposure of carcinogens.

The biliary duct trematode Platynosomum fastosum is considered a primary predisposing factor of cholangiocarcinoma in cats. P. fastosum was not observed in the two patients; although, the visualization of the adult parasite or its eggs by histopathological evaluation is not always possible. Another cause for the non-identification of the parasite could be that infections may have occurred in the past and thus have solved in the patients, although infections may cause chronic inflammation, which can culminate with e occurrence of the neoplasm $[1,4]$.

Among other predisposing factors of cholangiocellular carcinoma are the chemical agents plutonium and americium, intestinal parasites such as Ancylostoma spp. and Trichuris vulpis, and the presence of chronic inflammation independent of the etiology, as long as they cause exacerbated proliferation of biliary duct epithelium cells $[7,13]$.

Biliary duct carcinomas are firm, elevated, non-encapsulated, have a central umbilicated-like depression, and multiple whitish nodules [1,2,5-7], similar to that observed in cat 1 . Evaluation of cat 2 revealed tumors with the same consistency, but presented as masses of large sizes, covering almost all the hepatic parenchyma.

In the microscopic examination, the morphological findings led to the diagnosis of moderately differentiated metastatic cholangiocarcinoma, with metastases in the omentum (cat 1) and in the pancreas and lymph node (cat 2). This metastatic potential of cholangiocarcinoma was already evidenced in another study [1,2,5-7]. 


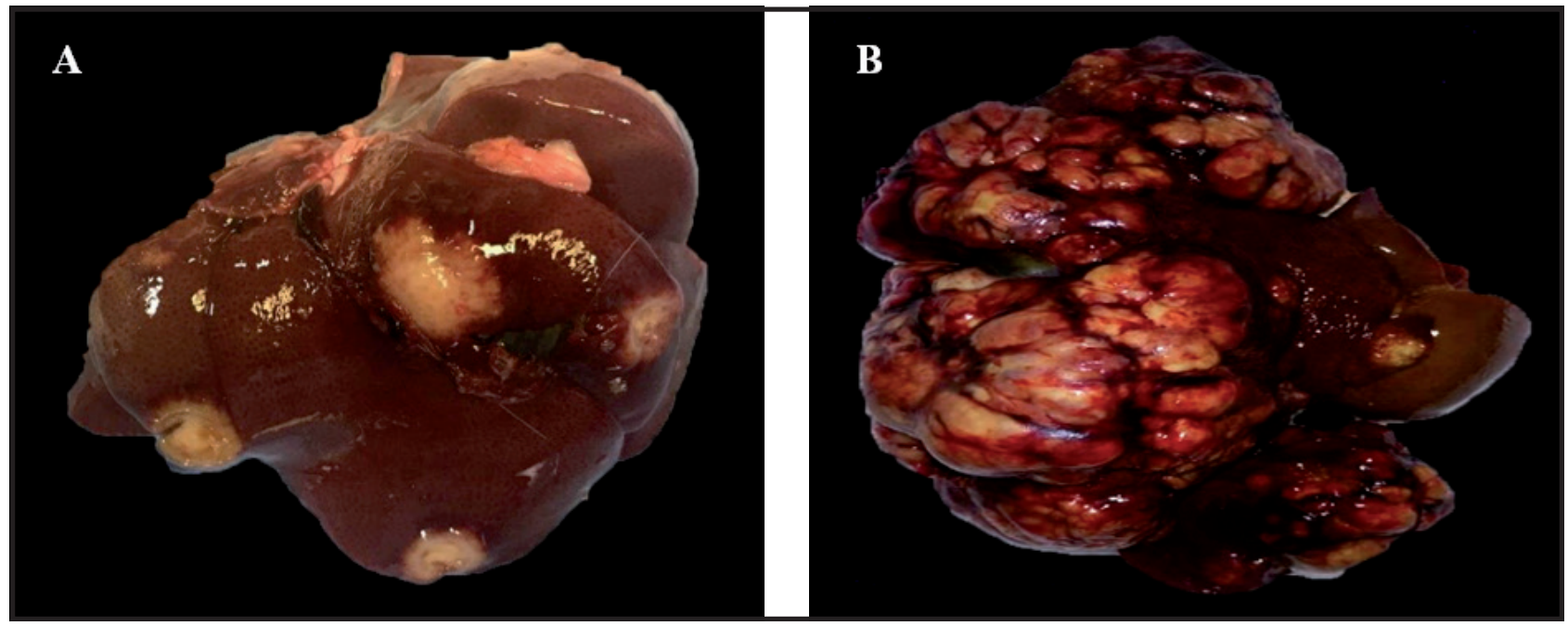

Figure 1. Hepatic parenchyma of two young felines with cholangiocarcinoma. A- Liver with nodules of whitish color, umbilicated, measuring up to $3.0 \mathrm{~cm}$ in diameter, randomly distributed by the parenchyma. B- Masses of whitish coloring covering the hepatic parenchyma in the parietal face.
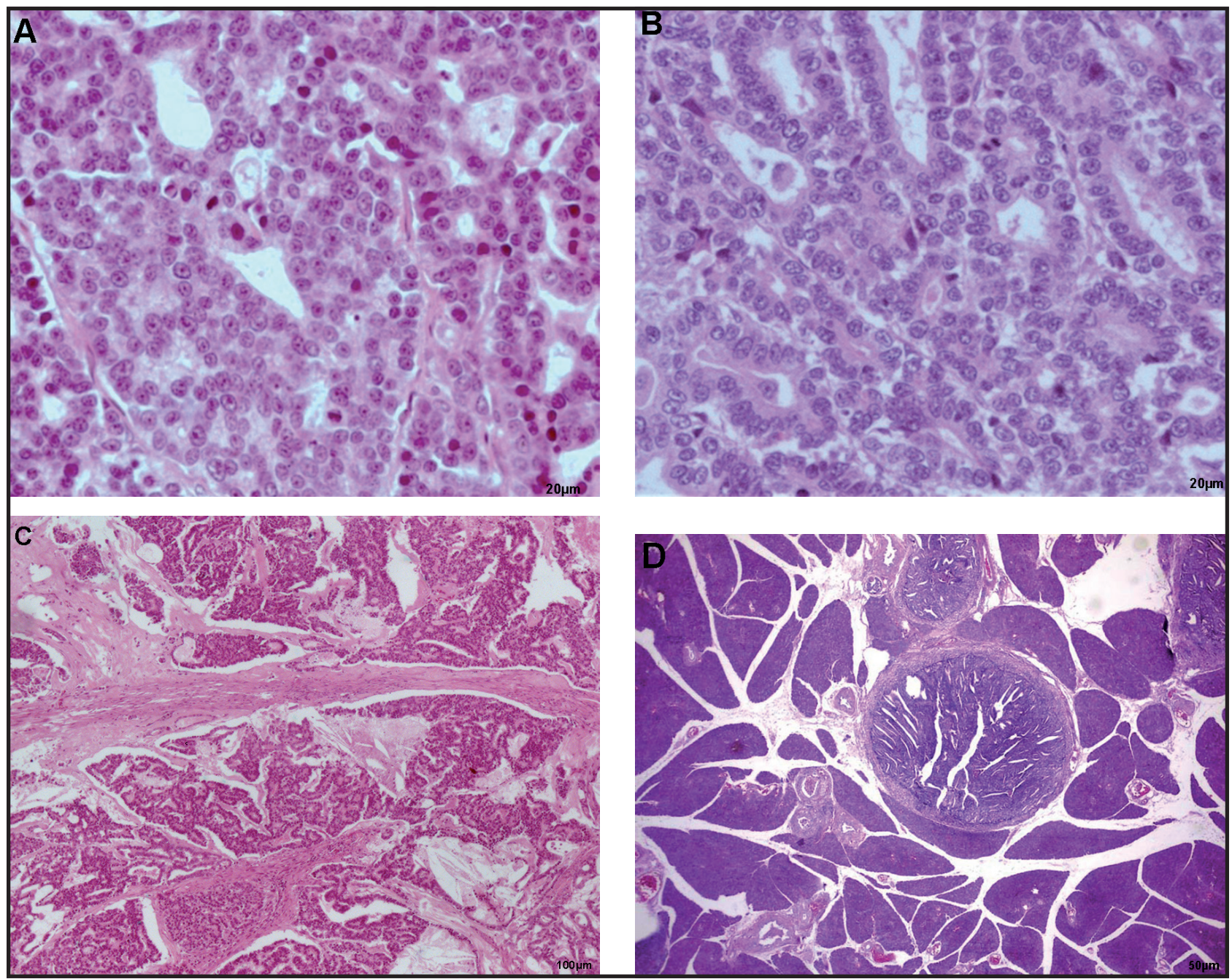

Figure 2. Diffuse proliferation of neoplastic cells of epithelial origin arranged in tubular form [HE; 63×obj.]. A- Microscopic analysis of cat 1. B- Microscopic analysis of cat 2. C- Neoplastic proliferation in the omentum of cat 1 (metastasis) [HE; 10xobj.]. D- Neoplastic proliferation in pancreas of cat 2 (metastasis) [HE; 20×obj.] 
Table 1. Immunohistochemical profile with markers for neoplastic cells and individual patient results.

\begin{tabular}{cccc}
\hline Antibodies & Clone & Neoplastic cells cat 1 & Neoplastic cells cat 2 \\
\hline $\begin{array}{c}\text { CK Pan- Intermediate epithelial cell filaments } \\
\text { CEA- Carcinoembryogenic antigen }\end{array}$ & AE1AE3 & Positive & Positive \\
$\begin{array}{c}\text { PK7- Marker of ductal, glandular, superficial and } \\
\text { transitional squamous epithelium }\end{array}$ & OV-TL12/30 & Positive & Positive \\
CK20- Marker of gastric epithelium, intestinal \\
$\begin{array}{c}\text { urothelium } \\
\text { TTF-1- Lung and thyroid carcinoma marker }\end{array}$ & Ks20.8 & Negative & Negative \\
$\begin{array}{c}\text { Pepatocyte specific antigen } \\
\text { CD10- Expressed in hepatocarcinomas and } \\
\text { renal carcinomas }\end{array}$ & OCH1E5 & Negative & Negative \\
5D56- Marker of neuroendocrine tumors & BC56C04 & Negative & Negative \\
CD56ative & Negative \\
\hline
\end{tabular}
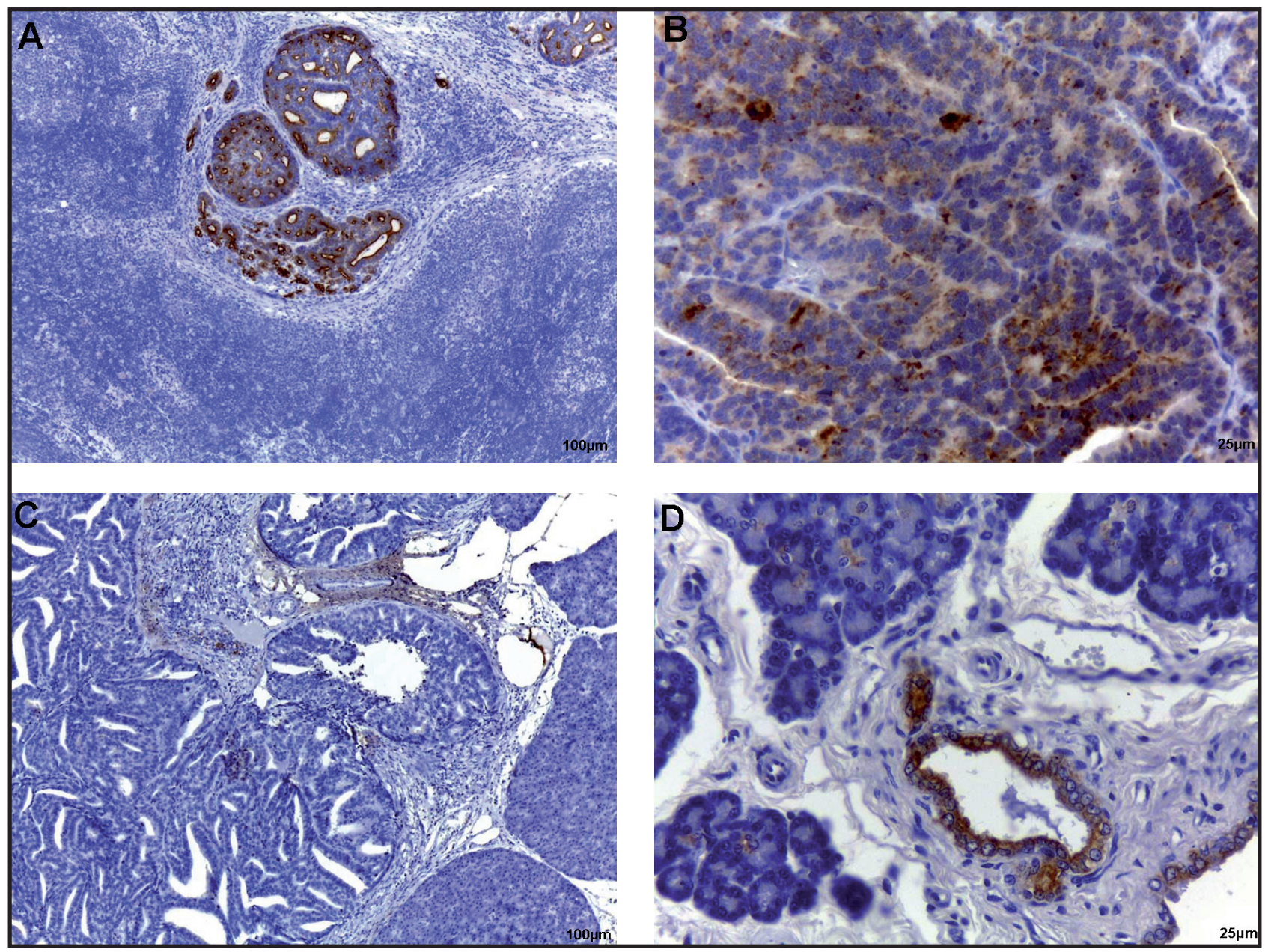

Figure 3. Immunohistochemical evaluation of two cats with biliary duct carcinoma. A- Positive marking for CK pan used to determine neoplastic cells in

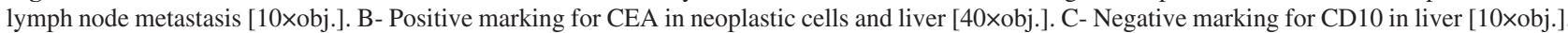
D- Negative marking for CK7 in neoplastic cells and positive for internal control [40×obj.]. 
Regarding immunohistochemistry, neoplastic cells were detected via the use of CK pan antibodies and CEA, such antibodies demonstrate immunoreactivity for most cholangiocarcinoma. The CEA antibodies are considered the most important tumor markers in differentiating cholangiocarcinoma from hepatocellular carcinoma [8-10]. The negative results for the hepatocyte-specific antigen and CD10 are in agreement with the studies in which these have been established as sensitive markers for hepatocellular carcinoma, with little expression in cholangiocarcinoma [9].

For individual analysis of the CK7 antibody, a negative result was obtained for each patient. These data differed from those observed in previous studies, where the expression of CK7 from moderate to high sensitivity and specificity is described for most cholangiocarcinoma in dogs (77.8\%) [12] and in humans (76\%) [9]. Scarce works demonstrate the efficacy of CK7 antibody in cats, which suggests that the utility of this marker may be limited in felines as well as verified in this study.

According to the authors' knowledge, this is the second report of cholangiocarcinoma in young cats, with only one study evidencing the presence of such neoplasia in a 4-year-old cat [2].

Clinical and anatomopathological observations made evident that these two young cats, with 3-year-old, had metastatic cholangiocarcinoma. Thus, veterinary providers should be aware that this tumor type can occur in young animals and be mindful of means of prevention and/or recommended treatment of predisposing factors.

Declaration of interest. The authors report no conflicts of interest. The authors alone are responsible for the content and writing of the paper.

\section{REFERENCES}

1 Andrade R.L.F.S., Dantas A.F.M., Pimentel L.A., Galiza G.J.N., Carvalho F.K.L., Costa V.M.M. \& Riet-Correa F. 2012. Platynosomum fastosum-induced cholangiocarcinomas in cats. Veterinary Parasitology. 190(1-2): 277-280.

2 Aslan O., Cakir L., Bekdik I.K., Dogan Z. \& Tunc A.S. 2014. Cholangiocarcinoma of intrahepatic bile ducts with disseminated metastases in a Siamese cat: a case report. Veterinarni Medicina. 59(7): 359-367.

3 Balkman C. 2009. Hepatobiliary neoplasia in dogs and cats. The Veterinary Clinics of North America. Small Animal Practice. 39(3): 617-625.

4 Braga R.R., Teixeira A.C., Oliveira J.A.A. \& Cavalcanti L.P.G. 2016. Prevalence of Platynosomum fastosum infection in free roaming cats in northeastern Brazil: Fluke burden and grading of lesions. Veterinary Parasitology. 309(227): 20-25.

5 Cunha V.A.F., Fernandes D.O., Bade P.L., Vieira-Filho C.H.C., Cunha V.A.F., Martins Filho E.F. \& Estrela-Lima A. 2016. Cistoadenocarcinoma biliar em felino - Relato de caso. Revista Brasileira de Medicina Veterinária. 38(1): 168-172.

6 Filgueira K.D., Reis P.F.C.C., Freitas V.A.L. \& Paula V.V. 2009. Colangiocarcinoma em felino doméstico: relato de um caso. Medvep: Revista Científica de Medicina Veterinária - Pequenos Animais e Animais de Estimação. 7(20): 203-207.

7 Gusmão M.A., Keller D., Santos Filho A.A. \& Torres M.B.A.M. 2015. Colangiocarcinoma em cão com metástase em omento, linfonodos regionais e pâncreas - relato de caso. Arquivos de Ciência Veterinária e Zoologia. 18 (2): 129-132.

8 acobs T.M. \& Snyder P.W. 2007. Mucinous cholangiocarcinoma in a cat. Journal of the American Animal Hospital Association. 43(3): 168-172.

9 Lau S.K., Prakash S., Geller S.A. \& Alsabeh R. 2002. Comparative immunohistochemical profile of hepatocellular carcinoma, cholangiocarcinoma, and metastatic adenocarcinoma. Human pathology. 33(12): 1175-1181.

10 Patnaik A.K. 1992. Morphologic and Immunocytochemical Study of Hepatic Neoplasms in Cats. Veterinary Pathology. 29(5): 405-415.

11 Patnaik A.K., Lieberman P.H., Erlandson R.A. \& Antonescu C. 2005. Hepatobiliary Neuroendocrine Carcinoma in Cats: A Clinicopathologic, Immunohistochemical, and Ultrastructural Study of 17 Cases. Veterinary Pathology. 42(3): 331-337.

12 Ramos-Vara J.A., Miller M.A. \& Johnson G.C. 2001. Immunohistochemical characterization of canine hyperplastic hepatic lesions and hepatocellular and biliary neoplasms with monoclonal antibody hepatocyte paraffin 1 and a monoclonal antibody to cytokeratin 7. Veterinary pathology. 38(6): 636-643.

13 Taylor G.N., LIoyd R.D., Mays, C.W., Angus W., Miller S.C., Shabestari L. \& Hahn F.F. 1991. Plutonium- or americium-induced liver tumors and lesions in beagles. Health Physics. 61(3): 337-347.

http://seer.ufrgs.br/ActaScientiaeVeterinariae

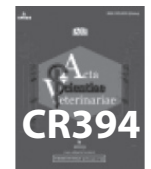

